\title{
Chaldäer im Platonismus
}

\section{Platon und die Weisheit der Alten}

„Griechenland ist groß, Kebes, und es gibt dort tüchtige Männer. Groß sind auch die Nationen der Barbaren, die ihr ... alle durchforschen müsst, und dabei dürft ihr weder Geld noch Mühen scheuen, gibt es doch nichts, wofür ihr euer Geld besser ausgeben könntet“ (Üb. Ebert). ${ }^{\mathrm{I}}$

Sokrates' Aufforderung ist in der Antike als Auftrag verstanden worden, für fremde Traditionen - gerade auch aus dem Orient - offen zu sein, fremde Völker aufzusuchen und sich belehren zu lassen. ${ }^{2}$ Platon selbst führt diese Offenheit in seinen Dialogen vor. An nicht wenigen Stellen beruft sich Sokrates auf Lehren der ,Alten“, auf Dichter oder Weise der Vorzeit - auch aus östlichen Ländern - und führt sie als Beleg für eigene - d.h. platonische - Lehren an, freilich nicht ohne sie auf eine Weise zu modifizieren und zu transformieren, so dass sie ins eigene Gedankengebäude passen. ${ }^{3}$ Denn die Dialoge zeigen auch: Die Bedeutung alter Weisheit wird zwar akzeptiert, dies jedoch nicht kritiklos und ungeprüft. Ein Automatismus „alter Logos gleich Wahrheit“ besteht für Platon nicht. Dieses kritische Rezeptionsverhältnis gilt für Platon, aber auch in anderen Bereichen des kulturellen Lebens seiner Zeit: Ein Satz aus der pseudo-platonischen Epinomis bringt es auf den Punkt. Demnach waren sich die Griechen bewusst, vieles der Weisheit der Barbaren, der barbaros philosophia wie es heißt, zu verdanken, aber auch dieses Übernommene zu schönerer Vollendung geführt zu haben. ${ }^{4}$ In dieser Haltung gegenüber der barbaros philosophia spiegelt sich jenes Selbstbewusstsein, das seit Marathon, Salamis und Plataiai im 5. Jahrhundert v. Chr. zu beobachten ist und das Christian Meier glücklich als „Könnensbewusst-

I Vgl. Platon, Phaidon 78a (T. Ebert, Phaidon. Übersetzung und Kommentar von T. Ebert, [= Platons Werke Bd. I 4], Göttingen 2004, 39); zur Stelle vgl. M. Erler, Kontexte der Philosophie Platons, in: Platon. Handbuch, hg. von C. Horn u.a., Stuttgart 2009, 6I-99, bes. 6Iff.; noch in der Spätantike wurde dieser Forschungsauftrag des Sokrates aufgegriffen, vgl. L.G. Westerink (Hg.), Olympiodorus Alexandrinus. Olympidori in Platonis Gorgiam commentaria, Leipzig I970, 4I, 6.

2 Vgl. M. Baltes, Der Platonismus und die Weisheit der Barbaren, jetzt in: M. Baltes, EPINOHMATA. Kleine Schriften zur antiken Philosophie und homerischen Dichtung, hg. v. M.-L. Lakmann, München/Leipzig 2005, I-26, bes. II; H. Dörrie, Platons Reisen zu fernen Völkern. Zur Geschichte eines Motivs der Platon-Legende und zu seiner Verwendung durch Lactanz, in: W. den Boer u.a. (Hgg.), Romanitas et Christianitas, Studia I.H. Waszink, Amsterdam/London I973, 99-II8.

3 Vgl. M. Erler, Legitimation und Projektion. Die ,Weisheit der Alten' im Platonismus der Spätantike, in:

D. Kuhn/H. Stahl (Hgg.), Die Gegenwart des Altertums, Heidelberg 200I, 313-326.

4 Vgl. Pseudo-Platonische Epinomis 986e. 
sein“ charakterisiert hat. 5 Es passt z. B. ins Bild, wenn Platon auf der Suche nach Vorbildern für die ideale Verfassung Athens im Dialog Timaios zunächst auf die alte ägyptische Stadt Sais verweist. Was freilich wie ein Beitrag ad maiorem gloriam Aegyptiorum aussieht, erweist sich schnell als Beleg für den Ruhm Athens. Denn jenes Sais in Ägypten galt Platon als eine Kopie des rooo Jahre zuvor gegründeten Ur-Athen. ${ }^{6}$ Hier kommt ein Selbstbewusstsein zum Ausdruck, das Offenheit gegenüber fremdem Wissen - vornehmlich aus dem Osten - mit souveräner Fähigkeit zu dessen Anverwandlung und Transformation verbindet. Schon Hesiod überträgt in den Werken und Tagen auf die Göttin Dike Vorstellungen östlichen, ,magischen‘ Königtums, wie sie u.a. in Ägypten, aber auch in Mesopotamien zu beobachten sind, indem er die Verehrung Dikes für das Wohlergehen von Mensch und Natur in der Polisgemeinschaft verantwortlich macht.7 Indem er jedoch diese Verehrung nicht mehr einer Person - dem König - anvertraut, wie dies in der ägyptischen oder in östlichen Gesellschaften der Fall war, sondern einem jeden Bürger, formuliert er mit Hilfe alter östlicher Vorstellungen etwas Neues und für das Verständnis griechischer Polisgemeinschaft Grundsätzliches: Jedes Mitglied trägt Verantwortung für ,Wohl und Weh' aller anderen Mitglieder und der Natur: In Sophokles' Oidipous Tyrannos wird deshalb nicht automatisch der König für jene Pest in Theben verantwortlich gemacht, mit der das Stück beginnt, sondern es wird der Verursacher unter allen Bürgern gesucht - man darf darüber spekulieren, ob im mesopotamischen oder ägyptischen Kontext ein „Oidipous Tyrannos“ wie der des Sophokles möglich gewesen wäre. ${ }^{8}$

\section{Babylon als Quelle alter Weisheit}

Als wohl wichtigste Quelle alter Weisheit galt Ägypten; doch auch Babylonien spielt eine wichtige Rolle, vor allem jenes Babylon, das uns schon früh in der griechischen Literatur begegnet. Der Bruder des Dichters Alkaios, Antimenidas, dient dort als Söldner. Babylon galt als bedeutende Metropole, die großen Mauern und die ,hängenden Gärten` als Weltwunder. Herodot berichtet von ihrer Größe und über seltsame Bräuche. ${ }^{9}$ Auch wenn seine Angaben wenig zuverlässig scheinen, - später wird manches von Ktesias zurechtgerückt und auch

5 Vgl. C. Meier, Die Entstehung des Politischen bei den Griechen, Frankfurt I983, 469ff.

6 Vgl. Platons Timaios 23d; dazu M. Baltes, Der Platonismus und die Weisheit der Barbaren, jetzt in: M. Baltes, EPINOHMATA. Kleine Schriften zur antiken Philosophie und homerischen Dichtung, hg. v. M.-L. Lakmann, München/Leipzig 2005, I2.

7 Vgl. dazu M. Erler, Das Recht (Dike) als Segensbringerin für die Polis. Die Wandlung eines Motivs von Hesiod zu Kallimachos, in: Studi Italiani di Filologia Classica 5 (1987), 5-36.

8 Vgl. M. Erler, Die drei Gebote des Aischylos im ,Oidipous Tyrannos' des Sophokles in: Würzburger Jahrbücher für die Altertumswissenschaft 28a (2004), 9-I9.

9 Vgl. Alkaios, Fragment 48. 50. 350 (Voigt, in: Sappho et Alcaeus. Fragmenta, hg. v. E.-M. Voigt, Amsterdam I963); dazu W. Burkert, Königs-Ellen bei Alkaios. Griechen am Rand der östlichen Monarchien, Journal of Medieval History 53/2 (I996), 69-72; vgl. Herodot I, I78ff. 
Aristophanes zeigt sich weniger beeindruckt, vielmehr macht er Babylon zum Gegenstand des Spottes - so zeigt dies alles doch: Babylon war Teil des literarischen Diskurses und Gegenstand des Interesses. ${ }^{\text {IO }}$ Es wurde zudem nicht nur wegen seines Reichtums und seiner Macht, sondern auch als Ort der Weisheit bewundert. Mit Babylon verband man Errungenschaften der Astronomie und Astrologie ebenso wie mit solchen der Psychologie, der Theologie und der Mathematik - denn der grundlegende Satz des Pythagoras war offenbar schon seit Jahrhunderten bei den Babyloniern im Gebrauch. ${ }^{\text {II }}$ Nach Pausanias stammt sogar die Lehre von der Unsterblichkeit der Seele von den Chaldäern und den indischen Magiern. ${ }^{\text {I2 }}$ Noch bei Macrobius findet sich eine Planetenordnung (Saturn, Jupiter, Mars, Sonne, Venus, Merkur, Mond), die nicht der platonischen (zu finden in Platons Politeia), sondern der chaldäischen Reihung folgt, die so auch bei Cicero und den Platonikern zu finden ist. ${ }^{\mathrm{I}}$ Rechentechniken hatten eine große Blüte erreicht und gingen wie später bei Pythagoras mit Zahlenmystik Hand in Hand - aber auch die Philosophie wird mit Babylon in Verbindung gebracht. ${ }^{I 4}$ Lukian lässt die personifizierte Philosophie dem Gott Zeus von ihrem Weg nach Griechenland berichten. ${ }^{15}$ Zuerst sei sie zu den Barbaren gegangen, $\mathrm{zu}$ den Brahmanen, den Indern und Äthiopiern, zu den Ägyptern und dann schließlich zu den Chaldäern und Magiern in Babylon. Noch Diogenes Laertios weiß von solchen, nach deren Meinung die Entwicklung der Philosophie ihren Anfang bei den ,Barbaren' genommen hat. ${ }^{16}$ Demnach hatten die Perser ihre Magier, die Babylonier und Assyrer ihre Chaldäer, und die Gallier ihre so genannten Druiden und Semnotheten. Es wundert vor diesem Hintergrund nicht, dass Babylon als ein Quell der barbaros philosophia zur Station jener Bildungsreise wurde, die man griechischen Weisen teilweise andichtete, um die Herkunft ihrer Weisheit zu erklären und die Wahrheit ihres Wissens zu legitimieren. Wir hören z.B., dass sich Pythagoras zunächst in Ägypten, danach aber zwölf Jahre in Babylon aufgehalten habe und dort von den Chaldäern, also den babylonischen Priestern, Unterricht in Mathematik, Astronomie, Astrologie, Musik und Traumdeutung erfahren sowie sich ihre Theologie und ihre Rituale angeeignet habe. ${ }^{17}$ Ähnliches hören wir von weiteren griechischen Weisen, darunter von

IO Ob in Aristophanes' Aves 549ff. und II24-38 wirklich eine Parodie der Beschreibung der Stadtmauer von Babylon wie bei Herodot vorliegt (vgl. Herodot I, I79), ist umstritten. Zu Ktesias' „Persika“ und seiner - durchaus problematischen - Herodotkritik vgl. F. Jacoby, s.v. Ktesias, RE II (I922), 2032-2073; vgl. auch F.W. König, Die Persika des Ktesias, Graz I972.

II Vgl. Diogenes Laertius 8, I2; dazu C. Riedweg, Pythagoras, München 2002, II9.

I2 Vgl. Pausanias 4, 32, 4 (Dörrie-Baltes 66.I, in: H. Dörrie/M. Baltes, Der Platonismus in der Antike Bd. 2, Stuttgart/Bad Cannstatt I990, I76 mit 454).

I3 Vgl. Platon, De re publica, 6I6d ff.; vgl. Cicero, De re publica, 6, I7 und Macrobius, In Somnium Scipionis 2, 3, I3; J. Flamant, Macrobe et le néo-platonisme latin à fin du IV siècle, Leiden I977, 42Iff.; H. Dörrie/M. Baltes, Der Platonismus in der Antike Bd. 6, 2, Stuttgart/Bad Cannstatt 2002, I9I.

I4 Vgl. C.H. Kahn, Pythagoras and the Pythagoreans. A Brief History, Indianapolis 200I, $342 \mathrm{f}$.

I5 Vgl. Lukian, fug. 6.

I6 Vgl. Diogenes Laertios I, I.

I7 Vgl. z.B. Porphyrius, Vita Pythagorae 6 (= Dörrie-Baltes 67, 6, in: H. Dörrie/M. Baltes, Der Platonismus in der Antike, Bd. 2, Stuttgart/Bad Cannstatt 1990, I82 mit 465). 
keinem geringeren als Platon. Freilich, Platon war so bedeutend, dass chaldäische Priester auch zu ihm pilgerten, wie Philipp von Opûs uns wissen lässt, dies freilich erst kurz vor seinem Tod, so dass er nicht mehr viel von ihnen lernen konnte. ${ }^{8}{ }^{8}$ Seneca zufolge sollen Magier in Athen dem Verstorbenen Platon geopfert haben. ${ }^{19}$ Gewiss, diese Geschichten sind zumeist Fiktion. Zudem protestiert der antike Philosophiehistoriker Diogenes Laertios gegen die Orientverbindung und lässt die Philosophie in Griechenland beginnen - eine Position, die besonders im I9. Jahrhundert der bedeutende Philosophiehistoriker Eduard Zeller wieder vehement vertritt, wenn er alles sich aus den „Voraussetzungen des griechischen Volkslebens entwickeln lässt““. ${ }^{20}$ Gleichwohl sind die Versuche, bedeutende Lehren mit der Weisheit des Ostens in Verbindung zu bringen und eigenen Errungenschaften auf diese Weise Autorität zu verleihen, ein Beleg für den Respekt, den die Griechen, insbesondere auch Philosophen der klassischen Zeit, dem Wissen der Alten entgegen gebracht haben und wie sehr sie es gewürdigt haben. ${ }^{2 \mathrm{I}}$ Babylon und Babylonien erweisen sich in diesem Kontext als wichtige Station und als Bezugspunkt - auch im Bereich der Philosophie.

\section{Homer und der Beginn der Philosophie}

Ein Beispiel für eine solche Beziehung lässt sich in der Tat sogar für den Anfang der griechischen Philosophie ausmachen und dies an prominenter Stelle des ersten Werkes europäischer Literatur, in Homers Ilias. Es handelt sich um eine Partie im Kontext jener berühmten Geschichte über den Betrug Heras an Zeus (der Dios Apate) im I4. Gesang. Hier hat die moderne Forschung Reminiszenzen mesopotamischer theologischer Vorstellungen erkannt. ${ }^{22}$

I8 Vgl. Philodem, Academicorum ind. col. 3, 36ff. (= K. Gaiser, Philodems Academica, Stuttgart/Bad Cannstatt I988, I76ff. mit 42Iff.; Dorandi, 3, 36ff., in: Filodemo, Storia dei Filosofi. Platone e l'Academia (PHerc. IO2I e I64), hg., übers. und komm. von T. Dorandi, Neapel I99I); Philipp von Opûs, Fragment 5 Tarán (in: L. Tarán, Academica. Plato, Philip of Opus and the Pseudo-Platonic Epinomis, Philadelphia I975); Fragment I4a Lasserre (in: F. Lasserre, De Léodamas de Thasos à Philippe d'Oponte, Neapel I987); dazu H. Dörrie, Die Wertung der Barbaren im Urteil der Griechen. Knechtsnaturen? Oder Bewahrer und Künder heilbringender Weisheit?, in: R. Stiehl/ A. Lehmann (Hgg.), Antike und Universalgeschichte, Festschrift für H.E. Stier, Münster I972, I46-I75; zu Platon vgl. M. Erler, Platon, in: Grundriss der Geschichte der Philosophie. Die Philosophie der Antike Bd. 2,2, hg. v. H. Flashar, Basel 2007, 48.

I9 Vgl. Seneca, Epistulae morales 58, 3I.

20 Vgl. E. Zeller, Die Philosophie der Griechen in ihrer geschichtlichen Entwicklung, Bd. I, 7. Auflage, Leipzig I923, 2I-52; interessant die „nota addizionale“ von R. Mondolfo in: E. Zeller/R. Mondolfo, La filosofia dei greci nel suo sviluppo storico, Bd. I,I, 3. Auflage, Florenz i959, 63-99.

2 I Vgl. Seneca, Epistulae morales 58, 3I; vgl. U. Hölscher, Anaximander und der Anfang der Philosophie, Hermes 8I (I953), 257-277. 385-4I8; erweiterte Fassung in: ders., Anfängliches Fragen, Göttingen I968, 9-89, dort 43; insbesondere M.L. West, The east face of Helicon. West Asiatic elements in Greek poetry und myth, Oxford I997; ders., Early Greek philosophy and the Orient, Oxford I97I; W. Burkert, Die Griechen und der Orient, München/Zürich 2003.

22 Vgl. W. Burkert, Die Griechen und der Orient, München/Zürich 2003, 36ff. 
In der berühmten Passage versucht Hera durch eine Täuschung Zeus vom Kampfgeschehen vor Troja abzulenken, weil dies sich so gar nicht nach ihren eigenen Vorstellungen und zum Nachteil der Griechen zu entwickeln scheint. ${ }^{23}$ Hera plant deshalb ein Schäferstündchen mit ihrem Gemahl. Zuvor freilich holt sie sich bei der zuständigen Göttin und Expertin in Liebesdingen, Aphrodite, taktischen Rat und Hilfe. Natürlich wäre es nicht zuträglich, wenn sie der anderen Göttin zu erkennen gäbe, wer das Opfer ihres Verführungsvorhabens sein soll. Hera ,improvisiert‘ deshalb eine Erklärung für ihren Wunsch, der edel klingt. Sie gibt vor, ihren Eltern, Okeanos und Tethys, helfen zu wollen. Sie sei deshalb auf dem Weg zu ihnen, die an den Grenzen der bewohnten Erde lebten:

„Denn ich mache mich auf zu den Grenzen der fruchtbaren Erde,

Will nach Okeanos sehn, dem Ahnen der Götter, und Tethys,

Die mich in ihrem Palast erzogen und hüteten, beide; [...]

Diese will ich besuchen, die dauernden Händel zu schlichten,

Denn schon lange Zeit vermeiden sie einer des andern

Ehelager und Liebe, getrennt durch bittere Feindschaft" (Üb. Rupé). ${ }^{24}$

Der Trug gelingt, und Hera kann Zeus mit Hilfe von Aphrodites Liebesmittel zu einem Schäferstündchen überreden.
„Also sprach der Kronide und schloß in die Arme die Gattin.
Unten erblühte die heilige Erde von sprießenden Gräsern,
Tauigem Lotosklee, Hyazinthen und saftigem Krokos,
Dicht und üppig und weich, die über den Boden sie hoben.
Beide lagerten dort und deckten sich zu mit den schönen
Goldenen Wolken; es fielen herab die Tropfen des Taues“ (Üb. Rupé). ${ }^{25}$

Diese Stelle ist wegen stilistischer Besonderheiten, die nicht so recht zum mündlichen Stil passen wollen, ${ }^{26}$ besonders aber aus inhaltlichen Gründen von Interesse. Okeanos und Tethys werden vorgestellt als Ursprung der Götter und Mutter und Okeanos als Ursprung für alles. Wenn es heißt, dass sich beide des Beilagers im Streit enthielten, wird signalisiert, dass das Werden - auch der Götter - zum Stillstand kommt. ${ }^{27}$ Zwar findet sich die Bemerkung im Kontext einer Trugrede. Doch bleibt der Eindruck, dass die Vereinigung von Zeus

23 Vgl. Homer, Ilias I4, I53 ff.

24 Vgl. Homer, Ilias I4, 200ff. Übersetzung: H. Rupé, Homer. Ilias, übertragen von dems., München I989, 477 .

25 Vgl. Homer, Ilias I4, 346ff. Übersetzung: H. Rupé, Homer. Ilias, übertragen von dems., München I989, 485. 26 Vgl. A. Dihle, Homer-Probleme, Opladen I970, 83-93.

27 Vgl. R. Cornford, A Ritual Basis for Hesiod's Theogony, in: The Unwritten Philosophy and other Essays, Cambridge I950, 95-II6; Vgl. W. Burkert, Die orientalisierende Epoche in der griechischen Religion und Literatur, Heidelberg I984, 7 ff. und ders., Die Griechen und der Orient, München/Zürich 2003, 57ff; Bezüge stellt bereits her O. Gruppe, Die griechischen Culte und Mythen in ihren Beziehungen zu den orientalischen Religionen, Leipzig $1887,614-622$. 
und Hera, die ein Aufblühen der Natur bewirkt, eine kosmische Dimension hat und geradezu Teil einer Kosmogonie wird. Diese Vorstellung ist Homers Anthropomorphismus ansonsten fremd und nur hier zu beobachten. Schon Gladstone hat dies I 890 erkannt, und nach ihm haben andere darauf hingewiesen, dass unsere Partie in der Tat enge Parallelen zu Wasserkosmogonien orientalischer Herkunft, insbesondere aber zum babylonischen Weltschöpfungsmythos „Enuma Elisch“ aufweist. Man denkt an Apsu - wie Okeanos ein Süßwassermeer, das die Welt umgibt und Untergrundquelle aller Quellen und Flüsse ist, der, wie es heißt, da war, als der Himmel oben und die Erde noch nicht existierten; man denkt an Tiamat, das Salzmeer als Gebärerin von allem. Wenn beide ihre Wasser vermischen, bringen sie die Götter hervor, den Himmel, und die Erde. ${ }^{28}$ Beide aber vermischten ihre Wasser bis Apsu eingeschläfert und Tiamat erschlagen wird. Weitere Entsprechungen im Kontext der Homerischen Dios Apate bestärken zudem den Eindruck, dass hier, am Beginn der griechischen Literatur, ein Echo dieses babylonischen Mythos zu vernehmen ist ein Echo, das nicht nur die literarische Darstellung Homers beeinflusst hat. Denn schon die Homerleser Platon ${ }^{29}$ und Aristoteles bringen Heras Hinweis auf Okeanos und Tethys mit den Anfängen der griechischen Philosophie, mit Thales, in Verbindung. In der Metaphysik des Aristoteles lesen wir:

„Manche meinen auch, dass auch die Alten, welche lange vor unserer Zeit und zuerst über die göttlichen Dinge geforscht haben, derselben Ansicht seien; denn den Okeanos und die Tethys machten sie zu Erzeugern der Entstehung ...“ und dann: „Thales jedoch soll sich auf diese Weise über die Grundursache ausgesprochen haben“ (Üb. Bonitz). ${ }^{\circ}$

Homer also als Kosmologe und Vorgänger des Vorsokratikers Thales. Dass Aristoteles bei Homer Theologisch-Philosophisches zu finden glaubt, kann nicht erstaunen, ist er doch der Meinung, dass Philosophie und Theologie schon in der Urzeit existiert haben, durch Sintfluten aber immer wieder in Vergessenheit geraten und nach der letzten Sintflut in rudimentärer mythischer Form tradiert worden seien, wie z. B. Kosmologien bei Autoren wie Orpheus, Homer, Hesiod oder Pherekydes aus Syros bezeugten. ${ }^{3}$ An eben jener Stelle also erkennt Aristoteles erste Ansätze der Philosophie, an der moderne Forschung ein Echo altorientalisch-babylonischer Vorstellungen ausgemacht hat. Auch bei Thales selbst hat man beobachtet, dass seine Vorstellungen - soweit wir das erkennen können mit solchen aus dem Orient, u.a. aus Babylonien konvergieren, aber auch, dass bei ihm entsprechende kosmologische Vorstellungen in mythologisch-anthropologischer Form

28 Vgl. W. Burkert, Die orientalisierende Epoche in der griechischen Religion und Literatur, Heidelberg I984, 88; vgl. Enuma Elisch I, Iff.; A. Heidel, The Babylonian Genesis, 2. Auflage, Chicago I95I; J.B. Pritchard, Ancient Near Eastern Texts Relating to the Old Testament, 3. Auflage, Princeton I969, 6r.

29 Vgl. Platon, Kratylos 402b (orphisches Gedicht), vgl. auch Platon, Timaios 40e (mit Unterschieden), dazu R. Janko, The Iliad. A Commentary, Books I3-I6, Bd. 4, Cambridge I992, I80ff.

30 Vgl. Aristoteles, Metaphysik 983b27; vgl. Aristoteles, Metaphysik. Übersetzt und herausgegeben von H. Bonitz u.a., Hamburg 1966, I6.

3I Vgl. Aristoteles, Metaphysik 1074a38ff. 
gleichsam ,physikalisch ' werden. ${ }^{32}$ Offenheit für Altes, verbunden mit Transformation also auch hier - Babylonisches am Beginn der griechischen Philosophie: Gewiss, Aristoteles wie auch Platon mag wohl kaum bewusst gewesen sein, dass Heras improvisierte Trugrede Bezüge zu babylonischen Vorstellungen erkennen lässt.

\section{Eudemos und Enuma Elisch}

Ein Schüler des Aristoteles jedoch, Eudemos aus Rhodos, der in der zweiten Hälfte des 4. Jahrhundert v. Chr. wirkte, stellt einen solchen Bezug her. In seinem Werk Geschichte der Theologie - einer Pionierarbeit der Religionsgeschichte - hat dieser Aristoteles-Schüler nämlich die Grundlagen der griechischen und der orientalischen Theologie dargestellt. Das Werk ist uns durch den verkürzten Bericht des Neuplatonikers Damaskios aus dem 5. Jahrhundert n. Chr. bekannt.33 Demnach ist Eudemos in seinem Werk bei der Darstellung von Prinzipienlehren vorphilosophischer, antiker Dichter offenbar chronologisch vorgegangen. Er hat Orpheus (bei dem die Nacht als Prinzip gilt), dann Homer mit Hinweis auf Okeanos und Tethys - d.h. unsere Stelle im I4. Gesang -, darauf Hesiod (mit dem Chaos), Akusilaos (auch mit dem Chaos), Epimenides (mit Aer und Nyx) und Pherekydes (mit Zeus und Chronos) behandelt. Bei Damaskios folgt sodann separat eine Besprechung der kosmologischen Prinzipien bei den Babyloniern, bei den persischen Magiern und den Ägyptern.

Moderne Interpreten haben nun darauf hingewiesen, dass sich Damaskios' Darstellung der babylonischen theologischen Prinzipienlehre konkret auf den Anfang des Enuma Elisch bezieht, wenn er nämlich von zwei Prinzipien spricht:34 Von Tauthe, der Mutter der Götter, und Apason; Tauthe entspricht dabei Tiamat, dem Salzwasser, Apson entspricht Apsu, dem Süßwasser. Die Stelle bei Damaskios wird deshalb gern als ein - freilich spätes Zeugnis für Kenntnisse babylonischer Mythologie im Griechischen herangezogen. Doch darf darauf hingewiesen werden, dass wir uns mit diesem Zeugnis keineswegs nur in der Philosophie der Spätantike bewegen. Ein Hinweis des Damaskios auf Eudemos macht nämlich deutlich, dass auch das Referat über babylonische kosmologische Vorstellungen schon von diesem stammt. Wir befinden uns also auch mit seinem Hinweis auf die Kos-

32 Vgl. z.B. die Sonnenfinsternis 586/5 v. Chr. nach Herodot I, 74, 9; zu Problemen dieser Stelle und zu babylonischen Vorbildern vgl. zu Thales als Astronom, M. L. Gemelli Marciano, Die Vorsokratiker Bd. I, Auswahl der Fragmente und Zeugnisse. Übersetzung und Erläuterungen von ders., Düsseldorf 2007, 22 ff. und zu Anaximander, U. Hölscher, Anaximander und der Anfang der Philosophie, Hermes 8I (I953), 257-277, 385-4I8.

33 Vgl. Eudemos, Fragment I50 Wehrli (in: F. Wehrli, Die Schule des Aristoteles. Text und Kommentar, Bd. 6-7, Basel I953) = Damascius I24 (in: Damascius Diadochus, dubitationes et solutiones in Platonis Parmenidem, Bd. I, hg. von C. A. Ruelle, Amsterdam I966); siehe dazu auch Damascius I23-I25 (ebenfalls bei Ruelle); zu Eudemos aus Rhodos und Babylon vgl. F. Wehrli u.a., Grundriss der Geschichte der Philosophie. Die Philosophie der Antike, Bd. 3. Peripatos, hg. von H. Flashar, 2. Auflage, Basel 2004, 558-563.

34 Vgl. Enuma Elisch I, 30-72; dazu W. Burkert, Die Griechen und der Orient, München/Zürich $2003,56$. 
mogonie des Enuma Elisch bereits in der Zeit des vierten bis dritten Jahrhundert v. Chr., jener Zeit also, in der wir ein generelles Interesse für fremde Kulturen im Frühhellenismus, nicht zuletzt infolge der Alexanderzüge und der mit ihnen verbundenen ,Globalisierung، vornehmlich bei Historikern, antreffen.

Gerade auch die Schule des Aristoteles folgte Sokrates' Aufforderung zur Offenheit gegenüber Fremdem und sammelte Material der barbaros philosophia. Theophrast und Straton z. B. haben in ihren Werken Über Erfindungen auch diese Barbaren berücksichtigt. 35 Dikaiarch behandelt neben den Ägyptern auch die Leistungen der Chaldäer; Hekataios von Abdera schreibt ein Buch mit dem Titel Über die Philosophie der Ägypter. Megasthenes verfasst Berichte über Indien, Ktesias setzt sich kritisch mit Herodots Bericht über Babylon auseinander und Berossos, ein Priester des Bel, verfasst ein Werk mit dem Titel Chaldaika in drei Bänden und widmet es Antiochos Soter - ein Werk, das wiederum als Quelle für Alexander Polyistor, Apollodoros und Juba diente. ${ }^{6}$ Auch in der Literatur wird Babylon zum Thema: In einem Roman des Chariton aus Aphrodisias, mit dem Titel Chaireas und Kallirhoe wird Babylon zu einem wichtigen Schauplatz des Geschehens.

\section{Diogenes aus Seleukeia am Tigris}

In der griechischen Philosophie begegnen wir zu dieser Zeit sogar bedeutenden Figuren, die aus Babylon oder dem Umland nach Griechenland kamen. Stellvertretend sei hier auf die Stoa und jenen Diogenes aus Seleukia hingewiesen, der auch der Babylonier genannt wurde, weil die Einwohner dieser Stadt der Landschaft den Namen gaben, wie wir bei Diogenes Laertios lesen.37 Jener Diogenes war Schüler des ersten Schulgründers Zenon, wurde dann Chrysipps Nachfolger in der Schulleitung der Stoa und nahm in hohem Alter gemeinsam mit Karneades und Kritolaos (I56/5 v. Chr.) an jener Philosophengesandtschaft teil, die in Rom in schwieriger politischer Lage für Athen gut Wetter machen sollte

35 Vgl. zu Aristoteles selbst, Aristoteles, De philosophia Fragment 9 Rose (in: V. Rose, Aristoteles Pseudepigraphus, Hildesheim/New York I97I) = Diogenes Laertios I,8 mit H. Flashar, Aristoteles. Fragmente zu Philosophie, Rhetorik, Poetik, Dichtung, übersetzt und erläutert von. H. Flashar/U. Dubielzig/B. Breitenberger, Berlin 2006, I33, wo Aristoteles anders als in der „Metaphysik“ die Anfänge der Philosophie u.a. bei den Magiern suchte; vgl. Aristoteles, De bon. Fragment 29 Rose (in dems.) = Diogenes Laertios I,I und dazu Aristoteles, De bono Fragment 30 (in dems.) = Diogenes Laertios I, 6-7 = Sotion, Fragment 36 Wehrli (in: F. Wehrli. Die Schule des Aristoteles. Texte und Kommentar, Supplement 2, Basel i978).

36 Vgl. Dikaiarch, Fragmente 55-58 Wehrli (in: F. Wehrli, Die Schule des Aristoteles. Texte und Kommentar, Bd. I, Basel I944); Hekataios von Abdera, FGrH 264 F 25 (in: F. Jacoby, Die Fragmente der griechischen Historiker, Bd. 3.I, Leiden I940); Berossos, FGrH 680 (in: F. Jacoby, Die Fragmente der griechischen Historiker, Bd. 3.CI, Leiden I958); zu Berossos vgl. P. Schnabel, Berossos und die babylonisch-hellenistische Literatur, Leipzig u.a. I923; S. Mayer Burstein, The Babyloniaca of Berossus, 2. Auflage, Malibu i980; G. P. Verbrugghe/J. M. Wickersham: Berossos and Manetho. Introduced and Translated. Native traditions in Ancient Mesopotamia and Egypt, Ann Arbor 1996.

37 Vgl. Diogenes Laertios 6, 8I. 
und in der Tat einen nachhaltigen Eindruck hinterließ. Mit Diogenes begegnen wir einem Stoiker, der diese Lehre vorsichtig weiterentwickelte, sich in Sprachphilosophie, vor allem aber in Ästhetik und insbesondere in der Musiklehre einen Namen machte. Denn er trat vehement für eine musisch-ethische und besonders für eine ,psychagogische‘ Funktion der Musik ein. Er unterschied bei ihr zwei Wahrnehmungsvermögen: Die Wahrnehmung der Sinneseindrücke und die Wahrnehmung der Lust oder Unlust, die diese Sinneswahrnehmung belgeitet. Mit letzterer erklärt er, warum es unterschiedliche, individuelle Empfindungen bei der Musik geben könne. Neben der Ästhetik setzte Diogenes auch in der Theologie eigene Akzente, insofern er monotheistische Aspekte stärkte. Wir sind gerade über seine Musiktheorie und ihre Bedeutung gut unterrichtet, weil sich der Epikureer Philodem intensiv und kritisch mit seiner Auffassung auseinandersetzte. Diese Ausführungen - und damit die Positionen des Diogenes - sind uns jetzt durch eine neue Edition mit verbesserten Lesungen in ihrer Bedeutung besser kenntlich..$^{8} \mathrm{Ob}$ man bei Diogenes Einflüsse jenes orientalisch-babylonischen Hintergrundes ausmachen kann, von dem er stammt, scheint zwar fraglich. Doch belegt die bedeutende Rolle, die ein Babylonier wie er im intellektuellen Leben Griechenlands spielen konnte, einmal mehr jene Offenheit, die Platons Sokrates einfordert, und die Rolle, die Babylonien dabei spielte. Jedenfalls wird immer wieder deutlich, dass Babylon auch in philosophicis keinen schlechten Namen hatte.

\section{Das positive Babylon-Bild im Platonismus der Kaiserzeit}

Babylon also als eine Quelle alter Weisheit: Nachdem im Hellenismus babylonisches Wissen durchaus gewürdigt worden war, begegnen wir in der Kaiserzeit einem sogar noch wachsenden Interesse an orientalischem Gedankengut - an ägyptischer Offenbarungsweisheit ebenso wie am babylonisch-chaldäischem Bereich. 39 Freilich weicht nun gerade in der Philosophie jene von Platon propagierte kritische Rezeptionshaltung einer Bereitschaft, ,altes Wissen‘ mit einer geradezu religiösen Verehrung zu verbinden und anzunehmen, eine Form der Rezeption, der es weniger um transformierende Anverwandlung als vielmehr um Legitimation des Eigenen mit Hilfe eines Rekurses auf die Autorität des Alten geht. $4^{\circ}$ Gerade im Platonismus der Kaiserzeit lässt sich dieser Umgang mit der barabaros philosophia beobachten. Programmatisch ist, was im 2. Jahrhundert n. Chr. der Platoniker

38 Vgl. Philodème de Gadara, Sur la musique Livre IV, hg., übersetzt und kommentiert von D. Delattre, Paris 2007, besonders Bd. 2, 9Iff.

39 Vgl. M. Baltes, Der Platonismus und die Weisheit der Barbaren, jetzt in: M. Baltes, EPINOHMATA. Kleine Schriften zur antiken Philosophie und homerischen Dichtung, hg. v. M.-L. Lakmann, München/Leipzig 2005, $8 \mathrm{ff}$.

40 Vgl. M. Erler, Legitimation und Projektion. Die ,Weisheit der Alten`im Platonismus der Spätantike, in: D. Kuhn/H. Stahl (Hgg.), Die Gegenwart des Altertums, Heidelberg 200I, 313-326. 
Kelsos aus Alexandrien in seiner Schrift Wahrer Logos, in der er Philosophie und Bildung der Hellenen gegen das Christentum verteidigte, ausführt. Kelsos spricht ausdrücklich von einem Urwissen bei den Alten, auch und vor allem bei barbarischen Völkern. Schon vor Platon verfügten demnach Völker wie die Ägypter, die Assyrer, die Perser oder die Inder u.a., über eine barbaros philosophia von höchster Autorität. ${ }^{\mathrm{I}}$ Denn jene weisen Männer und Völker verdankten ihre Weisheit göttlicher Inspiration. Sie waren einer derartig intuitiven Erkenntnis zugänglich, weil sie weniger verdorben waren als spätere Menschen, wie es heißt. Das Verdienst der Griechen habe dann darin bestanden, diese traditionelle Weisheit in systematischer Weise zu behandeln. Und wieder wird Mesopotamien, gerade auch Babylon, als Ort des Wissens gewürdigt. ${ }^{2}$

Merkmal der neuen, intensivierten Offenheit für alte Weisheit ist also die Suche nach Wahrheit und Erkenntnis, aber auch das Verlangen nach Autoritäten. Gerade im Platonismus dieser Zeit ist zu beobachten, dass jenes ,Könnensbewusstsein“ immer mehr abnimmt, welches Platon und noch später hellenistische Philosophen auszeichnete und das zu souveräner Offenheit, aber auch zu einer durchaus kritischen Anverwandlung fremden Wissens befähigte.

In der Kaiserzeit hingegen finden sich immer mehr Philosophen, die den früheren Optimismus, wonach der Mensch aus eigener Kraft Glück erlangen kann, nicht mehr teilen, sondern die Schwäche des Menschen konstatieren und von menschlicher Nichtigkeit sprechen. Das Bewusstsein des Unvermögens, sich aus eigener Kraft Wahrheit und Erkenntnis wenigstens annähern zu können, ist platonischen Philosophen der Kaiserzeit Anlass und Grund, sich religiösem Kult und sogar magisch-theurgischen Praktiken und Gebeten zu öffnen und hier Hilfe zu suchen.43 Da die Platoniker zudem kulturelle Gegebenheiten jedweder Epoche als Manifestation einer den Menschen von alter Zeit her zugeteilten und zugänglichen Wahrheit verstanden, erhielten im I. und 2. Jahrhundert n. Chr. alte religiöse Vorstellungen im Kontext der barbaros philosophia wachsenden Einfluss, nicht zuletzt wohl auch infolge der Konkurrenz mit dem wachsenden Einfluss des Christentums.

Eben in diesem Kontext treten Babylonier, genauer, jene Chaldäer in den Vordergrund, die als Fachleute der Astronomie, des Kalenderwesens und anderer Wissenschaften galten, deren Weltsicht von Determinismus und göttlicher Providenz geprägt war, die eng mit Orakelkunst in Verbindung gebracht wurden und die nicht zuletzt für die zeitweilige,

4I Vgl. Kelsos I, I4. I6 Bader (in: R. Bader, Der Alethes Logos des Kelsos, Stuttgart/Berlin I940, 44-45); vgl. dazu M. Frede, Celsus philosophus Platonicus, in: Aufstieg und Niedergang der römischen Welt, Bd. 2, 36, 2, Berlin/ New York 1994, 5183-5213, besonders 5194.

42 Vgl. Kelsos 7, 45 Bader und auch Kelsos I, 2 Bader (in: R. Bader, Der Alethes Logos des Kelsos, Stuttgart/Berlin I940).

43 Vgl. M. Erler, Hilfe der Götter und Erkenntnis des Selbst. Sokrates als Göttergeschenk bei Platon und den Platonikern, in: Th. Kobusch/M.Erler (Hgg.), Metaphysik und Religion. Zur Signatur spätantiken Denkens, München/Leipzig 2002, 387-4I4. 
intellektuelle Hegemonie Babylons verantwortlich waren. Wortorakel und Physiognomik erlaubten ihnen Aussagen über die Zukunft der Menschen und wurden mit ethischen Empfehlungen verbunden.44 Diese Chaldäer wurden infolge ihrer umfassenden Gelehrsamkeit und Reputation als Philosophen bezeichnet und als Lehrer späterer Philosophen angesehen - auf ihre Beziehung zu Platon hatte ich bereits hingewiesen.

Es ist kein Zufall, dass besonders die philosophisch-theologische Seite ihrer Lehre bei Platonikern starke Beachtung gefunden hat, und dass man glaubte, diese Aspekte in den sogenannten „chaldäischen Orakeln“ zu finden. 45

Im Bemühen der Platoniker, Symphonie zwischen theologischen und religiösen Anschauungen bei Griechen und Barbaren herzustellen, spielen die Chaldäer eine wichtige Rolle. ${ }^{6}$ Denn im kaiserzeitlichen Platonismus zählten die Chaldäer, die mit Assyrern gleichgesetzt wurden, als Quelle auch von religiöser Weisheit. Deshalb wurden insbesondere die chaldäischen Orakel gerne herangezogen und interpretiert. Porphyrios z.B. widmete der Auslegung dieser Orakel eine Schrift mit dem Titel Über die aus den Orakeln zu gewinnende Philosophie (De philosophia ex oraculis haurienda). ${ }^{47}$

Diese chaldäischen Orakel sind vermutlich im 2. oder 3. Jahrhundert n. Chr. von einem gewissen Julian, der den Beinamen ,der Theurge‘ trug, und seinem Vater, der ein Chaldäer war, verfasst worden. Diese Orakel sind also Produkte spätantiker Religiosität, stellen aber einen Bezug zur alten babylonischen Weisheit her. Deshalb trugen sie für die Platoniker der Zeit den Nimbus ,alten Wissens‘, wurden von ihnen auf ihrer Suche nach ,alter Weisheit‘ als hilfreiche Elemente ,alter Religiosität‘ empfunden und galten wie orphische Dichtung, homerische Hymnen oder Hesiods Gedichte als Zeugnisse von den Göttern eingegebener Weisheit. Neuplatonische Philosophen glaubten, in ihnen ein dem eigenen verwandtes philosophisches System zu erkennen, das auf diese Weise gleichsam göttliche Legitimation erfuhr. Man kann mit einigem Recht sagen, dass die Sammlung der chaldäischen Orakel gleichsam die Bibel des ausgehenden, platonisierenden Heidentums darstellen - an Bedeutung gleich dem platonischen Dialog Timaios. Auch hier ist in der Tat die Konkurrenzsituation zum Christentum greifbar. Man wollte ein ,Heiliges Buch des Heidentums der Bibel entgegensetzen..$^{8}$

Die ursprüngliche Sammlung der in Hexametern abgefassten Orakel ist freilich nicht erhalten. Einzelne Orakel sind uns in unterschiedlichen Kontexten überliefert, weil neuplatonische Philosophen sie eingehender Interpretation unterzogen. In der Tat stellen sie

44 Lukrez 5, $726 f$.

45 Vgl E. des Places, Oracles chaldaïques, Paris I97I; W. Kroll, De oraculis Chaldaicis, Breslauer philologische Abhandlungen, Bd. 7, I, Breslau I894, ND Hildesheim ig62; H. Lewy, Chaldaean Oracles and Theurgy, in: Recherches d' Archeologie, de Philologie et d' Histoire, Bd. I3, Le Caire I956.

46 Vgl. Athenaios I2, 529f. 530b.; Cicero, De divinatione I, 2, dazu W.J.W. Koster, Art. „Chaldäer“, in: Reallexikon für Antike und Christentum, Bd. 2 (I954), I006-IO2I, besonders IoIo-IOI3.

47 Porphyrius, De philosophia ex oraculis haurienda, hg. von G.G. Wolff, Hildesheim I962 (unveränderter reprographischer Nachdruck der Auflage Berlin 1856).

48 Vgl. Marinos, Vita Procli, hg. von J.F. Boissonade, Amsterdam I966, I70, I2. 
eine Art Heilslehre dar, in der orientalische Elemente mit platonischen Vorstellungen auf eine Weise verbunden sind, dass platonische Philosophen Grundlagen ihrer Philosophie wieder erkennen konnten. Denn in diesen Orakeln, die als Gottesgeschenke galten, fanden die Platoniker jene triadische Struktur der Realität (Vater oder erstem Intellekt, zweitem Intellekt und Seele) wieder, die im späteren Platonismus zum durchgehenden Strukturelement des Seins wird. Hier wie dort geht es um die Rettung der menschlichen Seele aus der Finsternis der sinnlichen Welt und ihre Rückführung zum Licht des geistigen Bereiches. Die Orakel handeln von Gott, der von der sinnlichen Welt getrennt ist, von dämonischen Wesen, die Göttliches und Sinnliches verbinden, von der Seele und ihrer Rückkehr in ihre geistige Heimat und vom Kosmos. Wenn sich die Seele an ihre Herkunft erinnert, kann sie sich vom körperlichen Bereich lösen und zum göttlichen Ursprung zurückkehren, wo sie befreit vom Zwang der Bestimmung Erlösung und Ruhe findet. Doch kann sie dies nach Überzeugung kaiserzeitlicher Platoniker eben nicht mehr allein aus eigener Kraft tun. Und eben hier machen die chaldäischen Orakel ihr Hilfsangebot: So hören wir49, dass der griechische Theologe Gregor aus Nazianz einerseits die Seele (noch) zum Göttlichen steigen lässt mit Hilfe der Vernunft. Die Chaldäer hingegen behaupteten, dass wir nur mit Hilfe materieller Riten zu Gott gelangen können. Eben diese aber bieten jene chaldäischen Orakel an, indem sie das Reden über Gott (Theologie) durch Einwirken auf Gottes Handeln (Theurgie) ergänzen und magische Praktiken für religiöse Aspekte funktionalisieren, um die Seele vom Körper zu befreien.

Auch wenn die chaldäischen Orakel also keine ,alten‘ Orakel sind, so zeigt doch ihre Bedeutung für kaiserzeitliche Platoniker die nachhaltige Wirkung, welche ,Babylon` oder ,Babylonisches‘ generell als Symbol für einen Hort, alten Wissens‘ behalten hat. Freilich, dieses ,Babylon` ist jetzt nicht mehr nur Quelle anregender Informationen, die der Prüfung bedürfen, sondern wird verstanden als Autoritäts- und Legitimationsinstanz, ja geradezu als Projektionsfläche, mit deren Hilfe man eigene Vorstellungen und deren Wahrheitsanspruch gleichsam sanktionieren zu können glaubte. Einer historisch analysierenden Sichtweise mag dieses Vorgehen, das für die Platoniker der Kaiserzeit bei ihrem Umgang mit alten Wissensinstanzen gängige Praxis war, problematisch scheinen. Doch ist zu bedenken, dass derartige Projektionen in dieser Zeit nicht zuletzt der Etablierung eigener Identität in einer schwierig gewordenen Welt, aber auch der Distanzierung gegenüber dem aufstrebenden Christentum dienten. Babylon als Hort ,alter Weisheit‘ steht in dezidiertem Kontrast zum Babylon-Bild der jüdisch-christlichen Tradition, in der Babylon durchweg negativ konnotiert ist. Das Alte Testament macht Babylon in der Erzählung vom Turmbau zum Symbol menschlicher Hybris und zum Begriff von Gottesfeindschaft..$^{\circ} \mathrm{Im}$

49 Vgl. Psellos, De Anima p. I32 O’Meara (D.J. O’Meara, Michaelis Pselli philosophica minora, Bd. 2, Leipzig I989, I-I64); dazu O. Geudtner, Die Seelenlehre der chaldäischen Orakel, Meisenheim am Glan I97I. Vgl. auch Proclus, Ek tes chaldaikes philosophias, hg. von A. Hahn, Halle I89ı.

50 Vgl. Genesis II,I,9; Jesaja I3, I9-22. 
christlichen Bereich wird die Stadt zum Decknamen für Rom und die negativ bewertete diesseitige Welt. Babylon als Hure, als Hort des Luxus und der Verkommenheit: Die Platoniker hatten eine klare Begründung für diese verfehlte Sichtweise. Diese hängt nach Ansicht der paganen Platoniker damit zusammen, dass Juden und Christen mit der Tradition aller alten Völker gebrochen hätten, seien doch die Juden von den Ägyptern, die Christen

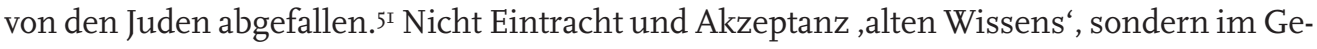
genteil Aufruhr (stasis) und Neuerungssucht (kainotomia) seien für ihr Verhalten kennzeichnend $5^{2}$ - also eine ganz andere Haltung als diejenige, die Platon und die späteren Platoniker propagierten und die ihre kritische Offenheit für Orientalisches begründete. Dieses Streben nach Neuerung machte dann offenbar den Weg frei für eine negative Sicht Babylons, als Quelle geistiger Fehlentwicklungen und Fehlverhaltens - eine Sichtweise, die sich auch da durchsetzte, wo ansonsten Platons Einfluss in anderer Hinsicht durchaus kenntlich blieb, wie Augustinus zeigt.53 Doch sollte darüber das positive Bild von Babylon nicht vergessen werden, das im pagan platonischen Kontext wirksam war.

5I Vgl. dazu Kelsos 8, 2 Bader (in: R. Bader, Der Alethes Logos des Kelsos, Stuttgart/Berlin i940), anders als z. B. Numenios sieht Kelsos den „Abfall“ und die Innovationssuche als Rezeptionshaltung der Juden und dann der Christen; M. Baltes, Der Platonismus und die Weisheit der Barbaren, jetzt in: M. Baltes EPINOHMATA. Kleine Schriften zur antiken Philosophie und homerischen Dichtung, hg. von M.-L. Lakmann, München/Leipzig $2005,6$.

52 Vgl. Kelsos 3, 5-I4. 7, 53. 8, 2 Bader (in: R. Bader, Der Alethes Logos des Kelsos, Stuttgart/Berlin I940), dazu M. Frede, Celsus philosophus Platonicus, in: Aufstieg und Niedergang der römischen Welt, Bd. 2, 36, 2, Berlin/ New York I994, 5195.

53 Vgl. Offenbarung I4, 8. I6, I9. I7, 5. I8, 2. I0. 21; B. Altaner, Art. „Babylon. D. Christlich“, in: Reallexikon für Antike und Christentum, Bd. I (I950), II30-II34; E. Lamirande, Art. „Babylon(ia)“, in: Augustinuslexikon, Bd. I (I986-I994), 566-569. 
